\title{
On a system of two diophantine inequalities with prime numbers
}

\author{
by \\ WenguANG Zhai (Jinan)
}

1. Introduction and results. In 1952 Piatetski-Shapiro [7] considered the following analogue of the Goldbach-Waring problem: Assume that $c>1$ is not an integer and let $\varepsilon$ be a small positive number. Let $H(c)$ denote the smallest natural number $r$ such that the inequality

$$
\left|p_{1}^{c}+\ldots+p_{r}^{c}-N\right|<\varepsilon
$$

is solvable in prime numbers $p_{1}, \ldots, p_{r}$ for sufficiently large $N$. Then it is proved in [7] that

$$
\limsup _{c \rightarrow \infty} \frac{H(c)}{c \log c} \leq 4 .
$$

Piatetski-Shapiro also proved that $H(c) \leq 5$ for $1<c<3 / 2$. In [8] Tolev first improved this result for $c$ close to one. More precisely, he proved that if $1<c<15 / 14$, then the inequality

$$
\left|p_{1}^{c}+p_{2}^{c}+p_{3}^{c}-N\right|<\varepsilon(N)
$$

has prime solutions $p_{1}, p_{2}, p_{3}$ for large $N$, where

$$
\varepsilon(N)=N^{-(1 / c)(15 / 14-c)} \log ^{9} N .
$$

This result was improved by several authors (see $[1,4,5])$.

In [9] Tolev first studied the system of two inequalities with primes

$$
\begin{aligned}
& \left|p_{1}^{c}+\ldots+p_{5}^{c}-N_{1}\right|<\varepsilon_{1}\left(N_{1}\right), \\
& \left|p_{1}^{d}+\ldots+p_{5}^{d}-N_{2}\right|<\varepsilon_{2}\left(N_{2}\right),
\end{aligned}
$$

where $1<d<c<2$ are different numbers and $\varepsilon_{1}\left(N_{1}\right)$ and $\varepsilon_{2}\left(N_{2}\right)$ tend to zero as $N_{1}$ and $N_{2}$ tend to infinity. Tolev proved that if $c, d, \alpha, \beta$ are real

1991 Mathematics Subject Classification: 11P55, 11D75.

This work is supported by National Natural Science Foundation of China (Grant No. 19801021) and Natural Science Foundation of Shandong Province (Grant No. Q98A02110). 
numbers satisfying

$$
\begin{aligned}
& 1<d<c<35 / 34, \\
& 1<\alpha<\beta<5^{1-d / c},
\end{aligned}
$$

then there exist numbers $N_{1}^{(0)}, N_{2}^{(0)}$, depending on $c, d, \alpha, \beta$, such that for all real numbers $N_{1}, N_{2}$ satisfying $N_{1}>N_{1}^{(0)}, N_{2}>N_{2}^{(0)}$ and

$$
\alpha \leq N_{2} / N_{1}^{d / c} \leq \beta,
$$

the system (1.3) has prime solutions $p_{1}, \ldots, p_{5}$ for

$$
\varepsilon_{1}\left(N_{1}\right)=N_{1}^{-(1 / c)(35 / 34-c)} \log ^{12} N_{1}, \quad \varepsilon_{2}\left(N_{2}\right)=N_{2}^{-(1 / d)(35 / 34-d)} \log ^{12} N_{2} .
$$

In this paper we shall prove

TheOREM. Suppose c and $d$ are real numbers such that

$$
1<d<c<25 / 24 \text {, }
$$

and $\alpha$ and $\beta$ are real numbers satisfying (1.5). Then for all real numbers $N_{1}, N_{2}$ satisfying (1.6), the system (1.3) has prime solutions $p_{1}, \ldots, p_{5}$ for

$$
\begin{aligned}
& \varepsilon_{1}\left(N_{1}\right)=N_{1}^{-(1 / c)(25 / 24-c)} \log ^{335} N_{1}, \\
& \varepsilon_{2}\left(N_{2}\right)=N_{2}^{-(1 / d)(25 / 24-d)} \log ^{335} N_{2} .
\end{aligned}
$$

A short proof, which follows the argument of Tolev [9], will be given in Section 2. The main difficulty is to prove the Proposition of Section 2, which improves Lemma 13 of Tolev [9] and is the key to our result. In Section 3, some preliminary lemmas are given. A detailed proof of the Proposition is given in Section 4. The new idea of the proof combines elementary methods and van der Corput's classical estimates.

Notations. Throughout, $c$ and $d$ are real numbers satisfying (1.7), $\alpha$ and $\beta$ are real numbers satisfying (1.5), and $\lambda$ denotes a sufficiently small positive number determined precisely by Lemma 1 of Tolev [9], depending on $c, d, \alpha, \beta . N_{1}$ and $N_{2}$ are large numbers satisfying (1.6). $X=N_{1}^{1 / c}$, $\varepsilon_{1}\left(N_{1}\right)=N_{1}^{-(1 / c)(25 / 24-c)} \log ^{335} N_{1}, \varepsilon_{2}\left(N_{2}\right)=N_{2}^{-(1 / d)(25 / 24-d)} \log ^{335} N_{2}$, $K_{1}=\varepsilon_{1}^{-1} \log X, K_{2}=\varepsilon_{2}^{-1} \log X, \eta$ is a sufficiently small positive number in terms of $c$ and $d, \tau_{1}=X^{3 / 4-c-\eta}, \tau_{2}=X^{3 / 4-d-\eta}, e(t)=e^{2 \pi i t}, \varphi(t)=e^{-\pi t}$, $\varphi_{\delta}(t)=\delta \varphi(\delta t)$, and $\chi(t)$ is the characteristic function of the interval $[-1,1]$. We set

$$
\begin{aligned}
B= & \sum_{\lambda X<p_{1}, \ldots, p_{5}<X} \log p_{1} \ldots \log p_{5} \chi\left(\frac{p_{1}^{c}+\ldots+p_{5}^{c}-N_{1}}{\varepsilon_{1} \log X}\right) \\
& \times \chi\left(\frac{p_{1}^{d}+\ldots+p_{5}^{d}-N_{2}}{\varepsilon_{2} \log X}\right),
\end{aligned}
$$




$$
\begin{gathered}
S(x, y)=\sum_{\lambda X<p<X}(\log p) e\left(x p^{c}+y p^{d}\right), \\
D=\int_{-\infty}^{\infty} \int_{-\infty}^{\infty} S^{5}(x, y) e\left(-N_{1} x-N_{2} y\right) \varphi_{\varepsilon_{1}}(x) \varphi_{\varepsilon_{2}}(y) d x d y, \\
\Omega_{1}=\left\{(x, y) \mid \max \left(|x| / \tau_{1},|y| / \tau_{2}\right)<1\right\}, \\
\Omega_{2}=\left\{(x, y) \mid \max \left(|x| / \tau_{1},|y| / \tau_{2}\right) \geq 1, \max \left(|x| / K_{1},|y| / K_{2}\right) \leq 1\right\}, \\
\Omega_{3}=\left\{(x, y) \mid \max \left(|x| / K_{1},|y| / K_{2}\right)>1\right\} .
\end{gathered}
$$

2. A short proof of the Theorem. The Theorem follows if we can show that $B$ tends to infinity as $X$ tends to infinity. By Lemma 3 of Tolev [9], it is sufficient to show that $D$ tends to infinity as $X$ tends to infinity. Write

$$
D=D_{1}+D_{2}+D_{3}
$$

where

$$
D_{i}=\iint_{\Omega_{i}} S^{5}(x, y) e\left(-N_{1} x-N_{2} y\right) \varphi_{\varepsilon_{1}}(x) \varphi_{\varepsilon_{2}}(y) d x d y .
$$

By the same arguments as in Section 4 of Tolev [9], we have

$$
D_{1} \gg \varepsilon_{1} \varepsilon_{2} X^{5-c-d} \text {. }
$$

By Lemma 4 of Tolev [9], we have

$$
D_{3} \ll 1 .
$$

So now the Theorem follows from (2.1)-(2.4) and the estimate

$$
D_{2} \ll \varepsilon_{1} \varepsilon_{2} X^{5-c-d}(\log X)^{-1} .
$$

By Lemma 14 of Tolev [9] we have

$$
\int_{-\infty}^{\infty} \int_{-\infty}^{\infty}\left|S^{4}(x, y)\right| \varphi_{\varepsilon_{1}}(x) \varphi_{\varepsilon_{2}}(y) d x d y \ll X^{2} \log ^{6} X .
$$

It suffices to prove the following

Proposition. Uniformly for $(x, y) \in \Omega_{2}$, we have

$$
S(x, y) \ll X^{11 / 12} \log ^{660} X .
$$

3. Some preliminary lemmas. In order to prove the Proposition, we need the following lemmas. Lemma 1 is Theorem 2.2 of Min [6]. Lemma 2 is Lemma 2.5 of Graham and Kolesnik [2]. Lemma 3 is contained in Lemma 2.8 of Krätzel [3]. Lemma 4 is well known (see Graham and Kolesnik [2], for example). 
Lemma 1. Suppose $f(x)$ and $g(x)$ are algebraic functions in $[a, b]$ and

$$
\begin{gathered}
\left|f^{\prime \prime}(x)\right| \sim 1 / R, \quad\left|f^{\prime \prime \prime}(x)\right| \ll 1 /(R U), \\
|g(x)| \ll G, \quad\left|g^{\prime}(x)\right| \ll G U_{1}^{-1}, \quad U, U_{1} \geq 1 .
\end{gathered}
$$

Then

$$
\begin{aligned}
\sum_{a<n \leq b} g(n) e(f(n))= & \sum_{\alpha<u \leq \beta} b_{u} \frac{g\left(n_{u}\right)}{\sqrt{\left|f^{\prime \prime}\left(n_{u}\right)\right|}} e\left(f\left(n_{u}\right)-u n_{u}+1 / 8\right) \\
& +O\left(G \log (\beta-\alpha+2)+G(b-a+R)\left(U^{-1}+U_{1}^{-1}\right)\right) \\
& +O(G \min (\sqrt{R}, 1 /\langle\alpha\rangle)+G \min (\sqrt{R}, 1 /\langle\beta\rangle)),
\end{aligned}
$$

where $[\alpha, \beta]$ is the image of $[a, b]$ under the mapping $y=f^{\prime}(x), n_{u}$ is the solution of the equation $f^{\prime}(x)=u$,

$$
b_{u}= \begin{cases}1 & \text { for } \alpha<u<\beta, \\ 1 / 2 & \text { for } u=\alpha \in \mathbb{Z} \text { or } u=\beta \in \mathbb{Z},\end{cases}
$$

and the function $\langle t\rangle$ is defined as follows:

$$
\langle t\rangle= \begin{cases}\|t\| & \text { if } t \text { is not an integer }, \\ \beta-\alpha & \text { otherwise, }\end{cases}
$$

where $\|t\|=\min _{n \in \mathbb{Z}}\{|t-n|\}$.

Lemma 2. Suppose $z(n)$ is any complex number and $1 \leq Q \leq N$. Then

$$
\left|\sum_{N<n \leq C N} z(n)\right|^{2} \ll \frac{N}{Q} \sum_{0 \leq q \leq Q}\left(1-\frac{q}{Q}\right) \operatorname{Re} \sum_{N<n \leq C N-q} z(n) \overline{z(n+q)} .
$$

Lemma 3. Suppose $f(x) \ll P$ and $f^{\prime}(x) \gg \Delta$ for $x \sim N$. Then

$$
\sum_{n \sim N} \min \left(D, \frac{1}{\|f(n)\|}\right) \ll(P+1)\left(D+\Delta^{-1}\right) \log \left(2+\Delta^{-1}\right) .
$$

Lemma 4. Suppose $5<A<B \leq 2 A$ and $f^{\prime \prime}(x)$ is continuous on $[A, B]$. If $0<c_{1} \lambda_{1} \leq\left|f^{\prime}(x)\right| \leq c_{2} \lambda_{1} \leq 1 / 2$, then

$$
\sum_{A<n \leq B} e(f(n)) \ll \lambda_{1}^{-1} .
$$

If $0<c_{3} \lambda_{2} \leq\left|f^{\prime \prime}(x)\right| \leq c_{4} \lambda_{2}$, then

$$
\sum_{A<n \leq B} e(f(n)) \ll A \lambda_{2}^{1 / 2}+\lambda_{2}^{-1 / 2} .
$$

Now we prove the following two lemmas, which are important in the proof of the Proposition. Let

$$
S=S\left(M, a, b, \gamma_{1}, \gamma_{2}\right)=\sum_{M<m \leq M_{1}} e\left(a m^{\gamma_{1}}+b m^{\gamma_{2}}\right),
$$


where $M$ and $M_{1}$ are positive numbers such that $5 \leq M<M_{1} \leq 2 M, a$ and $b$ are real numbers such that $a b \neq 0$, and $\gamma_{1}$ and $\gamma_{2}$ are real numbers such that $1<\gamma_{1}, \gamma_{2}<2, \gamma_{1} \neq \gamma_{2}$. Let $R=|a| M^{\gamma_{1}}+|b| M^{\gamma_{2}}$.

LEMMA 5. If $R M^{-1} \leq 1 / 8$, then

$$
S \ll M R^{-1 / 2} .
$$

Pro of. Suppose $R>100$; otherwise Lemma 5 is trivial. Let

$$
f(m)=a m^{\gamma_{1}}+b m^{\gamma_{2}} .
$$

Then

$$
f^{\prime}(m)=\gamma_{1} a m^{\gamma_{1}-1}+\gamma_{2} b m^{\gamma_{2}-1} .
$$

If $a b>0$, then $R / M \leq\left|f^{\prime}(m)\right| \leq 4 R / M \leq 1 / 2$, hence the assertion follows from Lemma 4.

Now suppose $a b<0$. Let

$$
\begin{aligned}
& I=\left\{t \in\left[M, M_{1}\right]|| f^{\prime}(t) \mid \leq R^{1 / 2} M^{-1}\right\}, \\
& J=\left\{t \in\left[M, M_{1}\right]|| f^{\prime}(t) \mid>R^{1 / 2} M^{-1}\right\} .
\end{aligned}
$$

By the definition we see that if $m \in J$, then

$$
R^{1 / 2} / M \leq\left|f^{\prime}(m)\right| \leq 4 R / M \leq 1 / 2 ;
$$

thus by Lemma 4 ,

$$
\sum_{m \in J} e(f(m)) \ll M R^{-1 / 2} .
$$

We only need to estimate $|I|$. If $t \in I$, then

$$
\begin{gathered}
\gamma_{1} a t^{\gamma_{1}}=-\gamma_{2} b t^{\gamma_{2}}+O\left(R^{1 / 2}\right)=-\gamma_{2} b t^{\gamma_{2}}\left(1+O\left(R^{-1 / 2}\right)\right), \\
t^{\gamma_{1}-\gamma_{2}}=\frac{-\gamma_{2} b}{\gamma_{1} a}\left(1+O\left(R^{-1 / 2}\right)\right),
\end{gathered}
$$

which implies that

$$
\begin{aligned}
t & =\left(\frac{-\gamma_{2} b}{\gamma_{1} a}\right)^{1 /\left(\gamma_{1}-\gamma_{2}\right)}\left(1+O\left(R^{-1 / 2}\right)\right)^{1 /\left(\gamma_{1}-\gamma_{2}\right)} \\
& =\left(\frac{-\gamma_{2} b}{\gamma_{1} a}\right)^{1 /\left(\gamma_{1}-\gamma_{2}\right)}\left(1+O\left(R^{-1 / 2}\right)\right) \\
& =\left(\frac{-\gamma_{2} b}{\gamma_{1} a}\right)^{1 /\left(\gamma_{1}-\gamma_{2}\right)}+O\left(M R^{-1 / 2}\right)
\end{aligned}
$$

So

$$
|I| \ll M R^{-1 / 2} .
$$

Now the conclusion follows from (3.1) and (3.3). 
Lemma 6. If $M \ll R \ll M^{2}$, then

$$
S \ll R^{1 / 2}+M R^{-1 / 3} .
$$

Proof. We have

$$
f^{\prime \prime}(m)=\gamma_{1}\left(\gamma_{1}-1\right) a m^{\gamma_{1}-2}+\gamma_{2}\left(\gamma_{2}-1\right) b m^{\gamma_{2}-2} .
$$

If $a b>0$, then $\left|f^{\prime \prime}(m)\right| \sim R M^{-2}$, and by Lemma 4 we get $S \ll R^{1 / 2}+$ $M R^{-1 / 2}$.

Now suppose $a b<0$. Let $\Delta_{0}=R^{2 / 3} M^{-2}$. Define

$$
\begin{aligned}
& I_{0}=\left\{t \in\left[M, M_{1}\right]|| f^{\prime \prime}(t) \mid \leq \Delta_{0}\right\}, \\
& I_{j}=\left\{t \in\left[M, M_{1}\right]\left|2^{j-1} \Delta_{0}<\right| f^{\prime \prime}(t) \mid \leq 2^{j} \Delta_{0} \leq 2 R / M^{2}\right\}, \\
& 1 \leq j \leq \frac{\log \left(\frac{2 R}{M^{2} \Delta_{0}}\right)}{\log 2}=J_{0} .
\end{aligned}
$$

If $I_{0}$ is not empty, then by the same argument as in Lemma 5 we get $\left|I_{0}\right| \ll M R^{-1 / 3}$. Thus Lemma 4 yields

$$
\begin{aligned}
\sum_{M<m \leq M_{1}} e(f(m)) & =\sum_{m \in I_{0}} e(f(m))+\sum_{1 \leq j \leq J_{0}} \sum_{m \in I_{j}} e(f(m)) \\
& \ll M R^{-1 / 3}+\sum_{1 \leq j \leq J_{0}}\left\{M\left(2^{j} \Delta_{0}\right)^{1 / 2}+\left(2^{j} \Delta_{0}\right)^{-1 / 2}\right\} \\
& \ll M R^{-1 / 3}+R^{1 / 2} .
\end{aligned}
$$

This completes the proof.

4. Proof of the Proposition. In this section we shall estimate $S(x, y)$ for $(x, y) \in \Omega_{2}$. Suppose $1<d<c<25 / 24$ and fix $(x, y) \in \Omega_{2}$. Let $R=|x| X^{c}+|y| X^{d}$. Obviously, $X^{3 / 4-\eta} \ll R \ll X^{25 / 24} \log ^{-300} X$. Without loss of generality, we suppose $x y \neq 0$. For the case $x=0$ or $y=0$, previous methods yield better results (see $[1,5]$ ).

Lemma 7. Suppose $a(m)$ are complex numbers such that

$$
\sum_{m \sim M}|a(m)|^{2} \ll M \log ^{2 A} M, \quad A>0 .
$$

Then for $M \ll \min \left(X^{2 / 3}, X^{19 / 12} R^{-1}\right), M N \sim X$, we have

$$
S_{\text {I }}=\sum_{m \sim M} a(m) \sum_{n \sim N} e\left(x(m n)^{c}+y(m n)^{d}\right) \ll X^{11 / 12} \log ^{A+1} X .
$$

Proof. If $M \ll X^{11 / 12} R^{-1 / 2}$, then by Lemma 6 we get

$$
S_{\mathrm{I}} \ll M\left(R^{1 / 2}+N R^{-1 / 3}\right) \log ^{A} X \ll X^{11 / 12} \log ^{A} X .
$$


From now on we always suppose $M \gg X^{11 / 12} R^{-1 / 2}$. Let $Q=\left[X^{1 / 6}\right]$. By Cauchy's inequality and Lemma 2 we have

$$
\begin{aligned}
\left|S_{\mathrm{I}}\right|^{2} & \ll \sum_{m \sim M}|a(m)|^{2} \sum_{m \sim M}\left|\sum_{n \sim N} e\left(x(m n)^{c}+y(m n)^{d}\right)\right|^{2} \\
& \ll X^{2} Q^{-1} \log ^{2 A} X+X Q^{-1} \log ^{2 A} X \sum_{q=1}^{Q}\left|E_{q}\right|,
\end{aligned}
$$

where

$$
\begin{gathered}
E_{q}=\sum_{m \sim M} \sum_{N<n \leq 2 N-q} e\left(x m^{c} \Delta(n, q ; c)+y m^{d} \Delta(n, q ; d)\right), \\
\Delta(n, q ; t)=(n+q)^{t}-n^{t} .
\end{gathered}
$$

Now the problem is reduced to showing that

$$
\sum_{q=1}^{Q}\left|E_{q}\right| \ll X \log ^{2} X .
$$

For each fixed $1 \leq q \leq Q$, let

$$
f(m, n)=x m^{c} \Delta(n, q ; c)+y m^{d} \Delta(n, q ; d) .
$$

We first consider several simple cases.

Case 0: A special case. For constants $a, b>0$, let $N(a, b)$ denote the solution of the inequality

$$
\left|a x(m n)^{c}+b y(m n)^{d}\right| \leq \frac{R}{Q^{1 / 2} \log X}, \quad m \sim M, n \sim N .
$$

Suppose $0<\sigma<1$ is a positive constant small enough. Then we can prove that uniformly for $a, b \in[\sigma, 1 / \sigma]$, we have

$$
N(a, b) \ll_{\sigma} X^{11 / 12} .
$$

If $x y>0$, then $N(a, b)=0$; so suppose $x y<0$. If $(m, n)$ satisfies the inequality (4.5), then

$$
\begin{aligned}
a x(m n)^{c} & =-b y(m n)^{d}+O\left(\frac{R}{Q^{1 / 2} \log X}\right) \\
& =-b y(m n)^{d}\left(1+O\left(Q^{-1 / 2} \log ^{-1} X\right)\right),
\end{aligned}
$$

which implies that 


$$
\begin{aligned}
m n & =\left(\frac{-b y}{a x}\right)^{1 /(c-d)}\left(1+O\left(Q^{-1 / 2} \log ^{-1} X\right)\right)^{1 /(c-d)} \\
& =\left(\frac{-b y}{a x}\right)^{1 /(c-d)}\left(1+O\left(Q^{-1 / 2} \log ^{-1} X\right)\right) \\
& =\left(\frac{-b y}{a x}\right)^{1 /(c-d)}+O\left(X Q^{-1 / 2} \log ^{-1} X\right)
\end{aligned}
$$

Thus (4.5) follows from a divisor argument. Why we study this case will be explained later.

CASE 1: $|\partial f / \partial m| \leq 500^{-1}$. It is obvious that

$$
\begin{aligned}
& \left|x m^{c} \Delta(n, q ; c)\right| \sim q|x| m^{c} n^{c-1} \sim q|x| X^{c} N^{-1}, \\
& \left|y m^{d} \Delta(n, q ; d)\right| \sim q|y| m^{d} n^{d-1} \sim q|y| X^{d} N^{-1},
\end{aligned}
$$

thus

$$
\left|x m^{c} \Delta(n, q ; c)\right|+\left|y m^{d} \Delta(n, q ; d)\right| \sim q R N^{-1} .
$$

We use Lemma 5 to estimate the sum over $m$ and get

$$
E_{q} \ll N M\left(q R N^{-1}\right)^{-1 / 2} \ll M N^{3 / 2} q^{-1 / 2} R^{-1 / 2} .
$$

Summing over $q$ we find that (4.4) holds if noticing $M \gg X^{11 / 12} R^{-1 / 2}$ and $R \ll X^{25 / 24}$.

CASE 2: $|\partial f / \partial n| \leq 500^{-1}$. For fixed $m$, we estimate the sum over $n$. Since

$$
\begin{gathered}
\partial f / \partial n=c x m^{c} \Delta(n, q ; c-1)+d y m^{d} \Delta(n, q ; d-1), \\
\Delta(n, q ; c-1)=(c-1) q n^{c-2}+O\left(q^{2} N^{c-3}\right), \\
\Delta(n, q ; d-1)=(d-1) q n^{d-2}+O\left(q^{2} N^{d-3}\right),
\end{gathered}
$$

we get

$$
\partial f / \partial n=c(c-1) x q m^{c} n^{c-2}+d(d-1) y q m^{d} n^{d-2}+O\left(q^{2} R N^{-3}\right) .
$$

If $x y>0$, then

$$
c_{1} q R N^{-2}<|\partial f / \partial n| \leq c_{2} q R N^{-2}<1 / 2
$$

for some constants $c_{1}, c_{2}>0$. Thus by Lemma 4 we get

$$
E_{q} \ll M N^{2} q^{-1} R^{-1}
$$

Now suppose $x y<0,0<\delta=o\left(q R N^{-2}\right)$ is a parameter to be determined. Define

$$
\begin{aligned}
& I=\{t \in[N, 2 N-q]|| \partial f / \partial t \mid \leq \delta\}, \\
& J=\{t \in[N, 2 N-q]|| \partial f / \partial t \mid>\delta\} .
\end{aligned}
$$


If $n \in I$, then we have

$$
\begin{aligned}
c(c-1) x q m^{c} n^{c-2} & =-d(d-1) y q m^{d} n^{d-2}+O\left(\delta+q^{2} R N^{-3}\right) \\
& =-d(d-1) y q m^{d} n^{d-2}\left(1+O\left(\delta N^{2}(q R)^{-1}+q N^{-1}\right)\right),
\end{aligned}
$$

which gives

$$
\begin{aligned}
n & =\left(\frac{-d(d-1) y m^{d}}{c(c-1) x m^{c}}\right)^{1 /(c-d)}\left(1+O\left(\delta N^{2}(q R)^{-1}+q N^{-1}\right)\right)^{1 /(c-d)} \\
& =\left(\frac{-d(d-1) y m^{d}}{c(c-1) x m^{c}}\right)^{1 /(c-d)}\left(1+O\left(\delta N^{2}(q R)^{-1}+q N^{-1}\right)\right) \\
& =\left(\frac{-d(d-1) y m^{d}}{c(c-1) x m^{c}}\right)^{1 /(c-d)}+\left(q+\delta N^{3} q^{-1} R^{-1}\right)
\end{aligned}
$$

Thus

$$
|I| \ll q+\delta N^{3} q^{-1} R^{-1} .
$$

By Lemma 4 we get

$$
\sum_{n \in J,|\partial f / \partial n| \leq 500^{-1}} e(f(m, n)) \ll \delta^{-1} .
$$

Thus we get

$$
\sum_{n \sim N,|\partial f / \partial n| \leq 500^{-1}} e(f(m, n)) \ll q+N^{3 / 2}(q R)^{-1 / 2},
$$

by choosing $\delta=(q R)^{1 / 2} N^{-3 / 2}$.

Combining the above, we get

$$
\sum_{\substack{(m, n) \\|\partial f / \partial n| \leq 500^{-1}}} e(f(m, n)) \ll M q+M N^{3 / 2}(q R)^{-1 / 2}+M N^{2}(q R)^{-1} .
$$

Summing over $q$ we find

$$
\begin{aligned}
& \sum_{q} \sum_{\substack{(m, n) \\
|\partial f / \partial n| \leq 500^{-1}}} e(f(m, n)) \\
& \ll M Q^{2}+M N^{3 / 2} Q^{1 / 2} R^{-1 / 2}+M N^{2} R^{-1} \log Q \ll X \log X,
\end{aligned}
$$

if we recall $X^{11 / 12} R^{-1 / 2} \ll M \ll X^{2 / 3}$.

CASE 3: For some $i$ and $j, 2 \leq i+j \leq 3$,

$(*)$

$$
\left|\frac{\partial^{i+j} f}{\partial m^{i} \partial n^{j}}\right| \leq \frac{q R \log X}{Q M^{i} N^{j+1}} .
$$


Let $c(\gamma, 0)=1, c(\gamma, n)=\gamma(\gamma-1) \ldots(\gamma-n+1)$ for $n \neq 0$. Then

$$
\begin{aligned}
\frac{\partial^{i+j} f}{\partial m^{i} \partial n^{j}}= & c(c, i) c(c, j) x m^{c-i} \Delta(n, q ; c-j) \\
& +c(d, i) c(d, j) y m^{d-i} \Delta(n, q ; d-j) .
\end{aligned}
$$

Since $c(c, i) c(c, j)$ and $c(d, i) c(d, j)$ always have the same sign, we may suppose $x y<0$; otherwise there is no $(m, n)$ satisfying $(*)$.

If $(m, n)$ satisfies $(*)$, then

$$
\begin{aligned}
c(c, i) c(c, j) x m^{c-i} \Delta(n, q ; c-j) & \\
= & -c(d, i) c(d, j) y m^{d-i} \Delta(n, q ; d-j)+O\left(\frac{q R \log X}{Q M^{i} N^{j+1}}\right) \\
& =-c(d, i) c(d, j) y m^{d-i} \Delta(n, q ; d-j)\left(1+O\left(\frac{\log X}{Q}\right)\right),
\end{aligned}
$$

which implies that

$$
\begin{aligned}
m & =\left(\frac{-c(d, i) c(d, j) y \Delta(n, q ; d-j)}{c(c, i) c(c, j) x \Delta(n, q ; c-j)}\right)^{1 /(c-d)}\left(1+O\left(\frac{\log X}{Q}\right)\right)^{1 /(c-d)} \\
& =\left(\frac{-c(d, i) c(d, j) y \Delta(n, q ; d-j)}{c(c, i) c(c, j) x \Delta(n, q ; c-j)}\right)^{1 /(c-d)}\left(1+O\left(\frac{\log X}{Q}\right)\right) \\
& =\left(\frac{-c(d, i) c(d, j) y \Delta(n, q ; d-j)}{c(c, i) c(c, j) x \Delta(n, q ; c-j)}\right)^{1 /(c-d)}+O\left(\frac{M \log X}{Q}\right) .
\end{aligned}
$$

Thus

$$
\sum_{(m, n),(*)} e(f(m, n)) \ll \frac{X \log X}{Q}
$$

and

$$
\sum_{q} \sum_{(m, n),(*)} e(f(m, n)) \ll X \log X .
$$

Now we turn to the most difficult part. We suppose that none of the conditions from Cases 0 to 3 holds. Without loss of generality, we suppose $\partial f / \partial n>0$. For any fixed $0 \leq j \leq(\log 10 Q) / \log 2$, let $I_{j}$ denote the subinterval of $[N, 2 N-q]$ in which

$$
\frac{2^{j} q R}{Q N^{3}}<\left|\frac{\partial^{2} f}{\partial n^{2}}\right| \leq \frac{2^{j+1} q R}{Q N^{3}} .
$$

We suppose $I_{j}=\left[A_{j}, B_{j}\right]$, say; $A_{j}$ and $B_{j}$ may depend on $m$, but this does not affect our final result. 
By Lemma 1 we get

$$
\sum_{n \in I_{j}} e(f(m, n))=e(1 / 8) \sum_{v_{1}(m)<v \leq v_{2}(m)} \frac{b_{v} e(s(m, v))}{\sqrt{|G(m, v)|}}+O(R(m, q, j))
$$

where

$$
\begin{aligned}
& f_{n}(m, g(m, v))=v, \\
& s(m, v)=f(m, g(m, v))-v g(m, v), \\
& G(m, v)=f_{n n}(m, g(m, v)), \\
& R(m, q, j)=\log X+\frac{Q N^{2}}{2^{j} q R}+\min \left(\frac{Q^{1 / 2} N^{3 / 2}}{2^{j / 2} q^{1 / 2} R^{1 / 2}}, \frac{1}{\left\|v_{1}(m)\right\|}\right) \\
& \quad \quad+\min \left(\frac{Q^{1 / 2} N^{3 / 2}}{2^{j / 2} q^{1 / 2} R^{1 / 2}}, \frac{1}{\left\|v_{2}(m)\right\|}\right), \\
& \frac{q R}{Q N^{2}} \ll v_{1}(m), v_{2}(m) \ll \frac{q R}{N^{2}} .
\end{aligned}
$$

Since

$$
\begin{gathered}
q R N^{-2} \gg 1, \\
v_{1}^{\prime}(m)=\frac{\partial^{2} f}{\partial n \partial m}\left(m, B_{j}\right) \gg q R Q^{-1} M^{-1} N^{-2}, \\
v_{2}^{\prime}(m)=\frac{\partial^{2} f}{\partial n \partial m}\left(m, A_{j}\right) \gg q R Q^{-1} M^{-1} N^{-2},
\end{gathered}
$$

by Lemma 3 we get

$$
\sum_{1 \leq q \leq Q} \sum_{j \geq 0} \sum_{m} R(m, q, j)
$$

$\ll \sum_{1 \leq q \leq Q} \sum_{j \geq 0}\left(M \log X+\frac{Q M N^{2}}{2^{j} q R}+\frac{q R}{N^{2}} \cdot \frac{Q^{1 / 2} N^{3 / 2}}{2^{j / 2} q^{1 / 2} R^{1 / 2}}+\frac{q R}{N^{2}} \cdot \frac{Q M N^{2}}{q R}\right)$

$\ll M Q^{2} \log ^{2} X+Q M N^{2} R^{-1} \log X+Q^{2} R^{1 / 2} N^{-1 / 2}$

$\ll X \log ^{2} X$.

Let $v_{1}=\min v_{1}(m), v_{2}=\max v_{2}(m)$. Then

$$
\sum_{M<m \leq 2 M} \sum_{v_{1}(m)<v \leq v_{2}(m)} \frac{b_{v} e(s(m, v))}{\sqrt{|G(m, v)|}} \ll \sum_{v_{1} \leq v \leq v_{2}}\left|\sum_{m \in I_{v}} \frac{e(s(m, v))}{\sqrt{|G(m, v)|}}\right|,
$$

where $I_{v}$ is a subinterval of $[M, 2 M]$.

Now the problem is reduced to estimating the sum over $m$. We first prove that $|G(m, v)|^{-1 / 2}$ is monotonic. Let $g=g(m, v)$. Differentiating the 
equation $f_{n}(m, g(m, v))=v$ over $m$ we get

$$
g_{m}(m, v)=-\frac{f_{n m}(m, g)}{f_{n n}(m, g)} .
$$

Thus

$$
G_{m}(m, v)=f_{m n n}+f_{n n n} g_{m}=\frac{f_{n n m} f_{n n}-f_{n n n} f_{n m}}{f_{n n}} .
$$

We only need to consider $f_{n n m} f_{n n}-f_{n n n} f_{n m}$, since $f_{n n}$ always has the same sign. Here we remark that we actually consider subintervals of $[M, 2 M]$ such that $f_{n n}$ is always positive or negative. This is so for other derivatives.

We now compute the corresponding derivatives. We have

$$
\begin{aligned}
f_{n m} & =c^{2} x m^{c-1} \Delta(g, q ; c-1)+d^{2} y m^{d-1} \Delta(g, q ; d-1) \\
& =c^{2}(c-1) x q m^{c-1} g^{c-2}+d^{2}(d-1) y q m^{d-1} g^{d-2}+O\left(\frac{q^{2} R}{M N^{3}}\right) .
\end{aligned}
$$

Since $\left|f_{n m}\right|>(q R \log X) /\left(Q M N^{2}\right)$, we have

$$
f_{n m}=\left(c^{2}(c-1) x q m^{c-1} g^{c-2}+d^{2}(d-1) y q m^{d-1} g^{d-2}\right)\left(1+O\left(\frac{Q^{2}}{N \log X}\right)\right) \text {. }
$$

Similarly,

$$
\begin{aligned}
f_{n n}= & \left(c(c-1)(c-2) x q m^{c} g^{c-3}+d(d-1)(d-2) y q m^{d} g^{d-3}\right) \\
& \times\left(1+O\left(\frac{Q^{2}}{N \log X}\right)\right), \\
f_{n n m}= & \left(c^{2}(c-1)(c-2) x q m^{c-1} g^{c-3}+d^{2}(d-1)(d-2) y q m^{d-1} g^{d-3}\right) \\
& \times\left(1+O\left(\frac{Q^{2}}{N \log X}\right)\right), \\
f_{n n n}= & \left(D(c) x q m^{c} g^{c-4}+D(d) y q m^{d} g^{d-4}\right)\left(1+O\left(\frac{Q^{2}}{N \log X}\right)\right),
\end{aligned}
$$

where $D(\gamma)=\gamma(\gamma-1)(\gamma-2)(\gamma-3)$.

For simplicity, we write $s=x m^{c} g^{c}, t=y m^{d} g^{d}$. Then we get

$$
\begin{aligned}
f_{n n} f_{n n m}- & f_{n m} f_{n n n} \\
& =m^{-1} g^{-6}\left(A s^{2}+2 B s t+C t^{2}\right)\left(1+O\left(\frac{Q^{2}}{N \log X}\right)\right),
\end{aligned}
$$

where

$$
\begin{aligned}
& A=c^{3}(c-2)^{2}(c-2)<0 \\
& B=c(c-1) d(d-1)\left(3 c d-c^{2}-d^{2}-c-d\right)<0, \\
& C=d^{3}(d-2)^{2}(d-2)<0
\end{aligned}
$$


We only need to show that

$$
A s^{2}+2 B s t+C t^{2} \neq 0
$$

If $x y>0,(4.19)$ is obvious. Now suppose $x y<0$. It is easy to show that $B^{2}-A C=c^{2}(c-1)^{2} d^{2}(d-1)^{2}(c-d)^{2}\left(2 c+2 d+1+c^{2}+d^{2}-4 c d\right)>0$.

Thus there exist constants $a_{1}, a_{2}, b_{1}, b_{2}$ such that

$$
A s^{2}+2 B s t+C t^{2}=\left(a_{1} s+b_{1} t\right)\left(a_{2} s+b_{2} t\right) .
$$

Since $A<0, B<0, C<0$, it can be easily seen that $a_{1} b_{1}>0, a_{2} b_{2}>0$. Now we recall that $s$ and $t$ do not satisfy the condition of Case 0. Taking $\sigma=\frac{1}{2} \min \left(\left|a_{1}\right|,\left|a_{2}\right|,\left|b_{1}\right|^{-1},\left|b_{2}\right|^{-1}\right)$ in Case 0, we obtain

$$
\left|a_{1} s+b_{1} t\right|>\frac{R}{Q^{1 / 2} \log X}, \quad\left|a_{2} s+b_{2} t\right|>\frac{R}{Q^{1 / 2} \log X} .
$$

Thus

$$
\left|A s^{2}+2 B s t+C t^{2}\right| \geq \frac{R^{2}}{Q \log ^{2} X} .
$$

This is the reason why we consider Case 0 .

By the above discussion we know that $|G(m, v)|$ is monotonic in $m$. So is $|G(m, v)|^{-1 / 2}$.

Now we compute $s_{m m}(m, v)$. We have

$$
\begin{aligned}
s_{m}(m, v) & =f_{m}(m, g)+f_{n}(m, g) g_{m}-v g_{m}=f_{m}(m, g), \\
s_{m m}(m, v) & =f_{m m}(m, g)+f_{m n}(m, g) g_{m}=\left(f_{m m} f_{n n}-f_{m n}^{2}\right) / f_{n n} .
\end{aligned}
$$

Similar to $G_{m}$, we have

$$
f_{m m} f_{n n}-f_{m n}^{2}=-\frac{2 q^{2}}{m^{2} n^{4}}\left(A_{1} s^{2}+B_{1} s t+C_{1} t^{2}\right)\left(1+O\left(\frac{Q^{2}}{N \log X}\right)\right),
$$

where $A_{1}=c^{3}(c-1)^{2}, B_{1}=c(c-1) d(d-1)(c+d), C_{1}=d^{3}(d-1)^{2}$, $B_{1}^{2}-4 A_{1} C_{1}>0$. Now if $x y>0$, we immediately get

$$
\left|f_{m m} f_{n n}-f_{m n}^{2}\right| \gg \frac{q^{2} R^{2}}{M^{2} N^{4}}
$$

if $x y<0$, then similar to $G_{m}$, we have

which implies

$$
\left|A_{1} s^{2}+B_{1} s t+C_{1} t^{2}\right| \gg \frac{R^{2}}{Q \log ^{2} X},
$$

$$
\left|f_{m m} f_{n n}-f_{m n}^{2}\right| \gg \frac{q^{2} R^{2}}{Q M^{2} N^{4} \log ^{2} X} .
$$

Combining the above, we get

$$
\left|s_{m m}\right| \gg \frac{q R}{Q M^{2} N \log ^{2} X} .
$$


On the other hand, we trivially have

$$
\left|s_{m m}\right| \ll\left|f_{m m}\right|+\left|f_{m n} g_{m}\right| \ll \frac{q R}{M^{2} N}+\frac{q R}{N^{2} M} \cdot \frac{N}{M} \ll \frac{q R}{M^{2} N} .
$$

Now let

$$
\begin{gathered}
I_{v, l}=\left\{\begin{array}{l|l}
m \in I_{v} \mid & \frac{2^{l} q R}{Q M^{2} N \log ^{2} X}<\left|s_{m m}\right| \leq \frac{2^{l+1} q R}{Q M^{2} N \log ^{2} X}
\end{array}\right\}, \\
0 \leq l \leq \log (Q \log X) / \log 2 .
\end{gathered}
$$

Then by partial summation and Lemma 4 we get

$$
\begin{aligned}
\sum_{q=1}^{Q} \sum_{j \geq 0} & \sum_{v=v_{1}}^{v_{2}}\left|\sum_{m \in I_{v}} \frac{e(s(m, v))}{\sqrt{|G(m, v)|}}\right| \\
& \ll \sum_{q=1}^{Q} \sum_{j \geq 0} \sum_{v=v_{1}}^{v_{2}} \sum_{l \geq 0}\left|\sum_{m \in I_{v}, l} \frac{e(s(m, v))}{\sqrt{|G(m, v)|}}\right| \\
& \ll \sum_{q=1}^{Q} \sum_{j \geq 0} \sum_{v=v_{1}}^{v_{2}} \sum_{l \geq 0}\left(\frac{Q N^{3}}{q R}\right)^{1 / 2} \\
& \times\left(M\left(\frac{2^{l} q R}{Q M^{2} N \log ^{2} X}\right)^{1 / 2}+\left(\frac{Q M^{2} N \log ^{2} X}{2^{l} q R}\right)^{1 / 2}\right) \\
& \ll \sum_{q=1}^{Q} \sum_{j \geq 0} \sum_{v=v_{1}}^{v_{2}}\left(\frac{Q N^{3}}{q R}\right)^{1 / 2}\left(\frac{(q R)^{1 / 2}}{N^{1 / 2}}+\frac{M\left(Q N \log ^{2} X\right)^{1 / 2}}{(q R)^{1 / 2}}\right) \\
& \ll \sum_{q=1}^{Q} \sum_{j \geq 0} \frac{q R}{N^{2}}\left(\frac{Q N^{3}}{q R}\right)^{1 / 2}\left(\frac{(q R)^{1 / 2}}{N^{1 / 2}}+\frac{M\left(Q N \log ^{2} X\right)^{1 / 2}}{(q R)^{1 / 2}}\right) \\
& \ll Q^{5 / 2} R N^{-1} \log ^{2} X+M Q^{2} \log ^{2} X \\
& \ll X \log ^{2} X,
\end{aligned}
$$

if we recall the condition $M \ll \min \left(x^{2 / 3}, x^{19 / 12} R^{-1}\right)$. This completes the proof of Lemma 7.

Lemma 8. Suppose $a_{m}$ and $b_{n}$ are complex numbers such that

$$
\sum_{m \sim M}\left|a_{m}\right|^{2} \ll M \log ^{2 A} M, \quad \sum_{n \sim N}\left|b_{n}\right|^{2} \ll N \log ^{2 A} N, \quad A>0, B>0 .
$$

Then for $X^{1 / 6} \ll N \ll \min \left(X^{3 / 2} R^{-1}, R X^{-1 / 3}\right)$, we have

$$
S_{\mathrm{II}}=\sum_{m \sim M} \sum_{n \sim N} a_{m} b_{n} e\left(x(m n)^{c}+y(m n)^{d}\right) \ll X^{11 / 12} \log ^{A+B+1} X .
$$


Proof. Take $Q=\left[X^{1 / 6} \log ^{-1} X\right]=o(N)$. Then by Cauchy's inequality and Lemma 2 again we get

$$
\begin{aligned}
\left|S_{\text {II }}\right|^{2} \ll & \frac{X^{2} \log ^{2 A+2 B} X}{Q} \\
& +\frac{X \log ^{2 A} X}{Q} \sum_{q=1}^{Q} \sum_{n}\left|b_{n} b_{n+q}\right|\left|\sum_{m \sim M} e(f(m, n))\right|,
\end{aligned}
$$

where $f(m, n)$ is defined as in the proof of Lemma 7 .

By Lemma 6 we get

$$
\sum_{m \sim M} e(f(m, n)) \ll q^{1 / 2} R^{1 / 2} N^{-1 / 2}+M N^{1 / 3} q^{-1 / 3} R^{-1 / 3} .
$$

Notice that for fixed $q$, we have

$$
\sum_{n}\left|b_{n} b_{n+q}\right| \ll \sum_{n}\left|b_{n}\right|^{2}+\sum_{n}\left|b_{n+q}\right|^{2} \ll N \log ^{2 B} N .
$$

The conclusion follows from the above three estimates.

Now we prove our Proposition. Let

$$
D=\min \left(X^{2 / 3}, X^{19 / 12} R^{-1}\right), \quad E=\min \left(X^{3 / 2} R^{-1}, R X^{-1 / 3}\right), \quad F=X^{1 / 6} .
$$

Then it is easy to check that under our assumptions we have

$$
D E>X, \quad X / D>(2 X)^{1 / 13}, \quad F^{2}<E .
$$

Using Heath-Brown's identity $(k=13)$ we know that $S(x, y)$ can be written as $O\left(\log ^{26} X\right)$ exponential sums of the form

$$
T=\sum_{n_{1} \sim N_{1}} \ldots \sum_{n_{26} \sim N_{26}} a_{1}\left(n_{1}\right) \ldots a_{26}\left(n_{26}\right) e\left(x\left(n_{1} \ldots n_{26}\right)^{c}+y\left(n_{1} \ldots n_{26}\right)^{d}\right),
$$

where

$$
\begin{aligned}
& N_{i}<n_{i} \leq 2 N_{i}(i=1, \ldots, 26), \quad X \ll N_{1} \ldots N_{26} \ll X, \\
& N_{i} \leq(2 X)^{1 / 13}(i=14, \ldots, 26), \\
& a_{1}\left(n_{1}\right)=\log n_{1}, \quad a_{i}\left(n_{i}\right)=1(i=2, \ldots, 13), \\
& a_{i}\left(n_{i}\right)=\mu\left(n_{i}\right)(i=14, \ldots, 26) .
\end{aligned}
$$

Some $n_{i}$ may only take value 1 . It suffices to show that for each $T$ we have

$$
T \ll X^{11 / 12} \log ^{630} X .
$$

We consider three cases.

CAse 1: There is an $N_{j}$ such that $N_{j} \geq X / D$. Since $X / D>X^{1 / 13}$, it follows that $1 \leq j \leq 13$. Without loss of generality, suppose $j=1$. Let $m=n_{2} n_{3} \ldots n_{26}, a_{m}=\sum_{m=n_{2} n_{3} \ldots n_{26}} \mu\left(n_{14}\right) \ldots \mu\left(n_{26}\right) \ll d_{25}(m), n=n_{1}$. 
Then $T$ is a sum of type I. By partial summation, Lemma 7 and a divisor argument we get

$$
T \ll X^{11 / 12} \log ^{630} X .
$$

CASE 2: There is an $N_{j}$ such that $F \leq N_{j}<X / D \leq E$. In this case we take $n=n_{j}, m=\prod_{i \neq j} n_{i}$. Then $T$ forms a sum of type II and (4.28) follows from Lemma 8.

CASE 3: $N_{j}<F(j=1, \ldots, 26)$. Without loss of generality, we suppose $N_{1} \geq \ldots \geq N_{26}$. Let $1 \leq l \leq 26$ be an integer such that

$$
N_{1} \ldots N_{l-1} \leq F, \quad N_{1} \ldots N_{l}>F .
$$

It is easy to check that $3 \leq l \leq 23$. We have

$$
F<N_{1} \ldots N_{l}=\left(N_{1} \ldots N_{l-1}\right) N_{l}<F^{2}<E .
$$

Let $n=n_{1} \ldots n_{l}, m=n_{l+1} \ldots n_{26}, a_{n}=\prod_{i=1}^{l} a_{i}\left(n_{i}\right), b_{m}=\prod_{i=l+1}^{26} a_{i}\left(n_{i}\right)$. Then $T$ forms a sum of type II and (4.28) follows from Lemma 8.

Now the Proposition follows from the above three cases.

Acknowledgements. Prof. Tolev kindly sent his papers to the author and the author is very grateful to him.

\section{References}

[1] Y. C. Cai, On a diophantine inequality involving prime numbers, Acta Math. Sinica 39 (1996), 733-742 (in Chinese).

[2] S. Graham and G. Kolesnik, Van der Corput's Method of Exponential Sums, London Math. Soc. Lecture Note Ser. 126, Cambridge Univ. Press, 1991.

[3] E. Krätzel, Lattice Points, Deutsch. Verlag Wiss., Berlin, 1988.

[4] A. Kumchev, A diophantine inequality involving prime powers, Acta Arith. 89 (1999), 311-330.

[5] A. Kumchev and T. Nedeva, On an equation with prime numbers, ibid. 83 (1998), $117-126$.

[6] S.-H. Min, The Methods of Number Theory, Science Press, Beijing, 1983 (in Chinese).

[7] I. I. Piatetski-Shapiro, On the distribution of prime numbers in sequences of the form $[f(n)]$, Mat. Sb. 33 (1953), 559-566 (in Russian).

[8] D. I. Tolev, On a diophantine inequality involving prime numbers, Acta Arith. 61 (1992), 289-306.

[9] - - On a system of two diophantine inequalities with prime numbers, ibid. 69 (1995), $387-400$.

Department of Mathematics

Shandong Normal University

Jinan, 250014, Shandong

P.R. China

E-mail: wgzhai@jn-public.sd.cninfo.net 